\title{
Energy loss of low-energy ions in transmission and backscattering experiments
}

\author{
D. Goebl, ${ }^{1}$ K. Khalal-Kouache, ${ }^{2}$ D. Roth, ${ }^{1}$ E. Steinbauer, ${ }^{1}$ and P. Bauer ${ }^{1}$ \\ ${ }^{1}$ Institut für Experimentalphysik, Abteilung für Atom- und Oberfächenphysik, Johannes Kepler Universität Linz, 4040 Linz, Austria \\ ${ }^{2}$ Université des Sciences et de la Technologie Houari Boumediene (USTHB), Faculté de Physique, Laboratoire SNIRM, Bab-Ezzouar, \\ 16111 Algiers, Algeria
}

(Received 14 May 2013; published 18 September 2013)

\begin{abstract}
The determination of the electronic stopping power for low-energy ions is an experimentally demanding task. In this paper we elaborate on the different effects of nuclear stopping and multiple scattering on the energy spectra for different experimental geometries, i.e., transmission through thin foils and backscattering from thin films. By calculating distributions of path lengths and scattering angles we demonstrate how electronic stopping, nuclear stopping, and multiple scattering add up to the total energy loss. We show that at low energies it is important to properly disentangle these effects to extract electronic stopping from the measured energy loss spectra.
\end{abstract}

DOI: 10.1103/PhysRevA.88.032901

PACS number(s): $34.50 . \mathrm{Bw}, 34.50 . \mathrm{Fa}$

\section{INTRODUCTION}

When ions traverse matter they lose energy along their trajectory. This energy loss has its origin in the interaction of the projectile with the nuclei and electrons of the target. Thus, it is common to distinguish between two different energy loss channels: energy loss in an elastic collision between projectile and target atoms is attributed to the nuclear stopping power $S_{n}$, while energy loss due to excitation of the electronic system of the projectile or target is described by the electronic stopping power $S_{e}$.

Recent years have seen an increasing interest in the determination of $S_{e}$ of solids for slow ions, i.e., at velocities $v$ well below the Fermi velocity, $v_{F}$. For a free electron gas (FEG) one would expect to find velocity proportionality of $S_{e}, S_{e}=$ $Q\left(Z_{1}, \mathrm{r}_{s}\right) v[1]$. The constant $Q$, usually referred to as "friction coefficient," is a function of the atomic number of the ion $Z_{1}$, and of the electron density of the FEG $n_{e}$, usually expressed in terms of the one-electron radius, $r_{s}=\left(3 / 4 \pi n_{e}\right)^{1 / 3}$, and has been modeled in detail [2,3]. Experiments revealed that electronic stopping of $\mathrm{H}$ and for He ions may exhibit a more sophisticated velocity dependence even when $v \ll v_{F}$, due to a more complex band structure of the conduction electrons in real metals [4-8] or in band gap materials [9-13]. Also charge exchange processes have been found to contribute efficiently to electronic energy loss [14-17].

Experimentally, there are various approaches to determine $S_{e}$, but at low ion velocities $\left(v \ll v_{F}\right)$ this is in any case a demanding task. In principle one can distinguish between measurements in backscattering geometry and transmission techniques, apart from determination of stopping powers from range profiles and other less common approaches like the inverted Doppler shift method [18]. Any energy loss measurement can only determine a total stopping power, $S$, which inevitably contains collisional losses to a certain extent; the detailed balance between elastic and inelastic

Published by the American Physical Society under the terms of the Creative Commons Attribution 3.0 License. Further distribution of this work must maintain attribution to the author(s) and the published article's title, journal citation, and DOI. losses depends on the scattering geometry and on experimental parameters.

In this investigation, we focus on experimental aspects of energy loss measurements at low velocities in a similar way as was done for proton stopping at the stopping maximum in Refs. [19] and [20]. We want to focus especially on the role of electronic and collisional losses in backscattering and transmission experiments to emphasize the challenges one meets in the determination of the electronic stopping power, $S_{e}$.

This paper is structured as follows: First, we discuss the principal mechanisms which contribute to the stopping power and their relevance for the different experimental techniques. Then we present simulations for the energy spectra obtained in transmission and backscattering experiments, where we disentangle the contribution of electronic and collisional losses to the total energy loss and discuss the relevance of multiple scattering in this context.

\section{TRANSMISSION VERSUS BACKSCATTERING}

In a transmission experiment (TR), the energy loss is determined by analyzing the energy spectrum of projectiles which are transmitted through a target of a certain thickness. For the sake of simplicity, we assume an experimental geometry where the incident beam and the detector are aligned along the surface normal of the target. In a typical backscattering experiment (BS) electronic stopping is determined by analyzing the energy spectrum of backscattered projectiles. In contrast to TR, only projectiles which are scattered by a certain scattering angle $\theta$ $>90^{\circ}$ are detected. Here we will focus on backscattering from thin films. There is, however, also the possibility to deduce information on electronic stopping from thick samples [21].

For high ion energies or very low target thicknesses, it is possible to deduce electronic stopping using simple models, which neglect the influence of plural or multiple scattering on the experimental data. In this case, electronic stopping can be determined in a straightforward way, as shown in Fig. 1: In TR, the energy loss $\Delta \mathrm{E}$ is exclusively due to electronic stopping, and $\Delta E=S_{e}(\langle E\rangle) \Delta x$ holds; the finite width of the peak is entirely due to electronic straggling. In BS, the trajectories consist of straight lines on the ways in and out, respectively, 


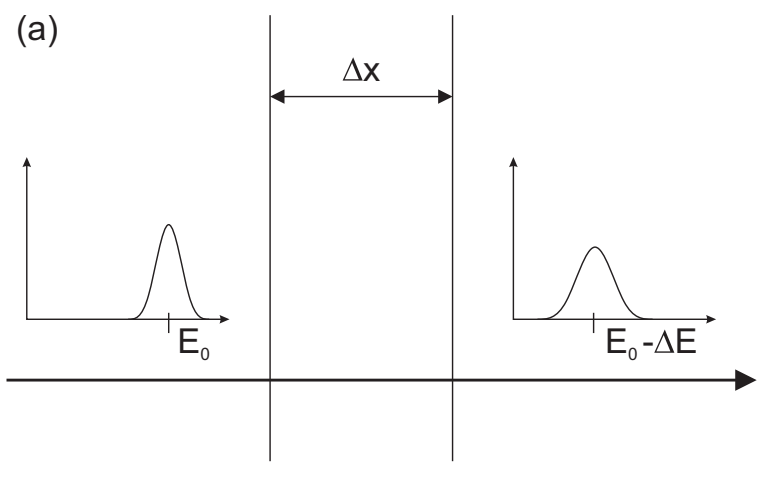

(b)

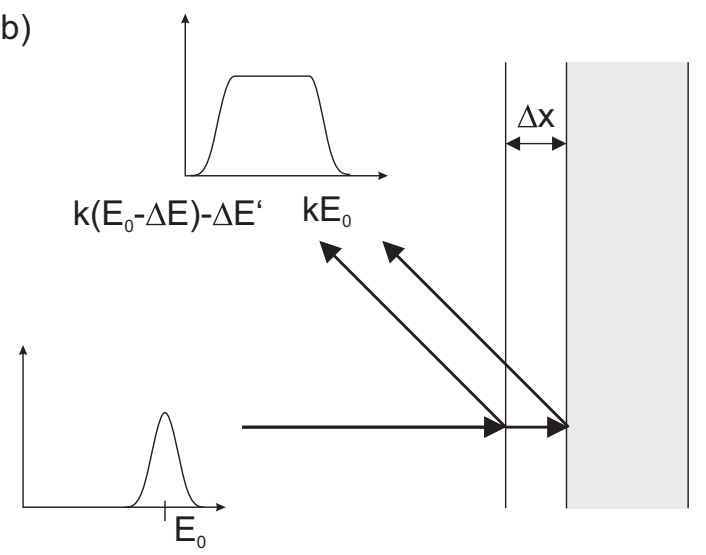

FIG. 1. Schematic of transmission (a) and backscattering (b) experiments. $E_{0}$ denotes the primary energy, the energy loss in a foil or thin layer of thickness $\Delta x$ is represented by $\Delta E ; k$ is the kinematic factor.

which intersect at the point where the backscattering collision takes place; the collisional energy loss is considered by the so-called kinematic factor $k$, which depends on the masses and on $\theta$. In this case, the width of the peak in the backscattering spectrum is related to the energy losses along the ingoing and outgoing paths, $\Delta E_{\text {in }}$ and $\Delta E_{\text {out }}$, respectively [22].

At low energies the probability for elastic scattering is considerably high such that plural or multiple scattering cannot be neglected $a$ priori. Therefore, the evaluation of experiments at lower energies is a more complex task:

(a) Nuclear stopping: at low velocities, $S_{n}$ may contribute significantly to the total stopping power and, in some cases, even exceed $S_{e}$. Note that $S_{n}$ refers to the mean energy loss occurring at all possible scattering angles, $0 \leqslant \theta \leqslant \pi$. Therefore, in any experiment with restricted acceptance angle, the energy loss experienced by the projectiles is due to an effective nuclear stopping power $S_{n}^{*}<S_{n}$. Consequently, in TR the effective nuclear stopping will contribute less than in BS, $S_{n, \mathrm{TR}}^{*}<S_{n, \mathrm{BS}}^{*}$. Different models have been presented to determine the effective contribution of nuclear losses in different geometries [23].

(b) Electronic stopping: since in a solid the electron density is nonuniform, impact parameter dependence in electronic stopping is observed for certain experimental conditions, e.g., in channelling geometry [24-26]. When, however, trajectories comprise a multitude of scattering events, e.g., in polycrys- talline targets, one may assume that electronic energy loss along the trajectory is similar in TR and BS. The question remains about the influence of electronic energy loss in violent atomic collisions, which are present only in BS. Experiments in the MEIS regime have shown that for single crystals the electronic energy loss is considerably increased for the single scattering peak in double alignment geometry, i.e., for collisions with small impact parameters [26]. This finding was explained by correlation of atom positions along the outgoing path and enhanced probabilities for inner shell excitations. Certainly, one has to expect similar effects to occur also in LEIS in double-alignment geometry, but to a lesser extent, since slow ions can excite only valence or conduction electrons. For polycrystalline materials, no correlation between the atom positions along the trajectory can be expected. Consequently, enhanced electronic energy loss should not occur due to spatial correlation. In the backscattering collision, impact parameter dependent inelastic losses may lead to an energy loss larger than anticipated from the kinematic factor and stopping power. When the width of a backscattering spectrum is evaluated, all contributing projectiles have experienced similar violent collisions; therefore, the width of the spectrum should not depend significantly on impact parameter dependent inelastic losses. Note that for He scattered from polycrystalline $\mathrm{Cu}$ it was possible to quantitatively reproduce the energy spectrum in a wide energy range including the surface peak by a Monte Carlo (MC) simulation, which includes only multiple elastic collisions and electronic stopping along the trajectory [27]. It is, however, still an open question whether impact parameterdependent inelastic losses are responsible for the observed major discrepancies between electronic stopping data deduced from TR and BS experiments [4,6,7,28]. In this context it is interesting that around the stopping maximum electronic stopping data are consistent within experimental uncertainties $( \pm 3 \%)$ when obtained in transmission and backscattering geometries [29].

In addition, preequilibrium charge effects may be noticeable for very thin samples or high velocities. Even when charge equilibrium is obtained, charge exchange processes and excitation of projectile electrons contribute to the electronic stopping power at sufficiently low velocities [14,30]. Charge exchange processes, such as reionization of neutral He, may require a minimum distance between the collision partners [31], resulting in a dependence of the energy loss on the impact parameter. On this basis, it was explained why in some materials the velocity dependence of the electronic stopping power is more complex for He ions than for $\mathrm{H}$ ions [16].

(c) Multiple scattering: another aspect of atomic collisions is that scattering at large impact parameters (small scattering angles) leads to a broadening of the angular distribution of the beam along the path. The resulting angular spread is characterized by the characteristic half width angle, $\psi_{1 / 2}$, which depends on the atomic numbers $Z_{1}, Z_{2}$, ion energy $E$ and target thickness $\Delta x[32]$ :

$$
\psi_{1 / 2} \sim Z_{1} Z_{2} \Delta x / E
$$

This angular spread leads to increased path lengths of the projectiles, with the mean path length depending on the experimental geometry. The relative influence of path length 
increase on the measured energy loss is expected to be more pronounced in BS than in TR.

In the following we distinguish between the scattering angle in individual scattering events, $\psi$, which add up to the transmission angle $\alpha$, or the backscattering angle $\theta$, respectively. Furthermore, one may distinguish between plural scattering (PS) and multiple scattering (MS). An intuitive way to do so is to consider collisions with $\psi<\psi_{1 / 2}$ as MS, and with $\psi>\psi_{1 / 2}$ as PS. Note, however, that, especially for large $\psi$, this definition is not meaningful anymore, since per definition, MS is related to small scattering events only.

In backscattering from a thin film, PS and MS lead to substantial modifications of the shape of a backscattering spectrum [33-35]:

(1) At energies above the high-energy edge, $k E_{0}$, a highenergy background is observed.

(2) The width of the backscattering spectrum will be enlarged due to increased mean path length and additional elastic losses.

(3) The low-energy edge will be blurred since at larger depth the identity $\psi \equiv \theta$ does not hold anymore.

(4) At energies below the low-energy edge, a background is observed ("low-energy background").

(5) Due to contributions from PS and MS, the height of the backscattering spectrum will be increased with increasing scattering depth.

At the high-energy edge, $k E_{0}$, however, the spectrum height will be dominated by trajectories which have undergone only one scattering event by a large angle [36].

Compared to BS, the energy spectra of transmitted projectiles will be influenced by PS and MS to a lesser extent. This can be understood in terms of scattering probabilities, since scattering events at large impact parameters $b$ occur with higher probabilities than those at small $b$. In TR, the idealized straight line trajectories would correspond to infinitely large impact parameters; PS and MS will therefore lead to large deviations from the straight line only with low probability. In contrast, in BS also in the idealized case the probability of the backscattering collision is low. Therefore, PS and MS lead to an increase of the scattered intensity by allowing for combinations of several scattering events. Consequently, in transmission the main modification of the energy loss spectrum is due to MS, i.e., due to large impact parameter collisions. This leads to the following consequences:

(1) The broadening of the angular distribution of the beam along the path will lead to a path length distribution $N(L, \alpha)$, depending on path length $L$ and exit angle $\alpha$. From this $N(L$, $\alpha$ ) distribution, a most probable path length $\hat{L}(\alpha)$ and the mean path length $\langle L\rangle(\alpha)$ may be deduced.

(2) $\hat{L}(\alpha)$ and $\langle L\rangle(\alpha)$ correspond to a most probable electronic energy loss $\Delta \hat{E}_{e}(\alpha)$ and a mean electronic energy $\operatorname{loss}\left\langle\Delta E_{e}\right\rangle(\alpha)$.

(3) In addition, the PS and MS events also lead to elastic energy losses, $\Delta E_{n}^{*}(\alpha)$.

For direct transmission, $\alpha=0$, the influences of these effects will be minimized, their relative importance depending on $\psi_{1 / 2}$ and on the total energy loss [37]. Possible contributions due to foil thickness variations or roughness will not be considered here [38,39].
In general for both TR and BS, any measured total energy loss, $\Delta \mathrm{E}_{\text {tot,TR }}$ and $\Delta \mathrm{E}_{\mathrm{tot}, \mathrm{BS}}$, comprises contributions due to a multitude of different processes, which are enumerated above. Therefore, it is necessary to take into account the path length increase and contributions due to nuclear stopping if one wants to deduce electronic stopping from the measured spectra.

\section{HOW TO DEDUCE ELECTRONIC STOPPING FROM THE TOTAL ENERGY LOSS}

In order to deduce electronic stopping from measured total energy losses, MC simulations are helpful in determining the relative importance of the individual processes. In TR and in $\mathrm{BS}$, different MC codes are applied, even if both programs belong to the transport of ions in matter (TRIM) family and are based on very similar physical models. Note that these simulations are valid only for targets of well-defined density, but without crystalline structure.

For TR geometry, TRIM-T2D [40] and an enhanced version [41] of TRIM-85 [42] were used to determine the energy loss distribution $N\left(\Delta E_{\mathrm{tot}}\right)$. In these programs electronic stopping

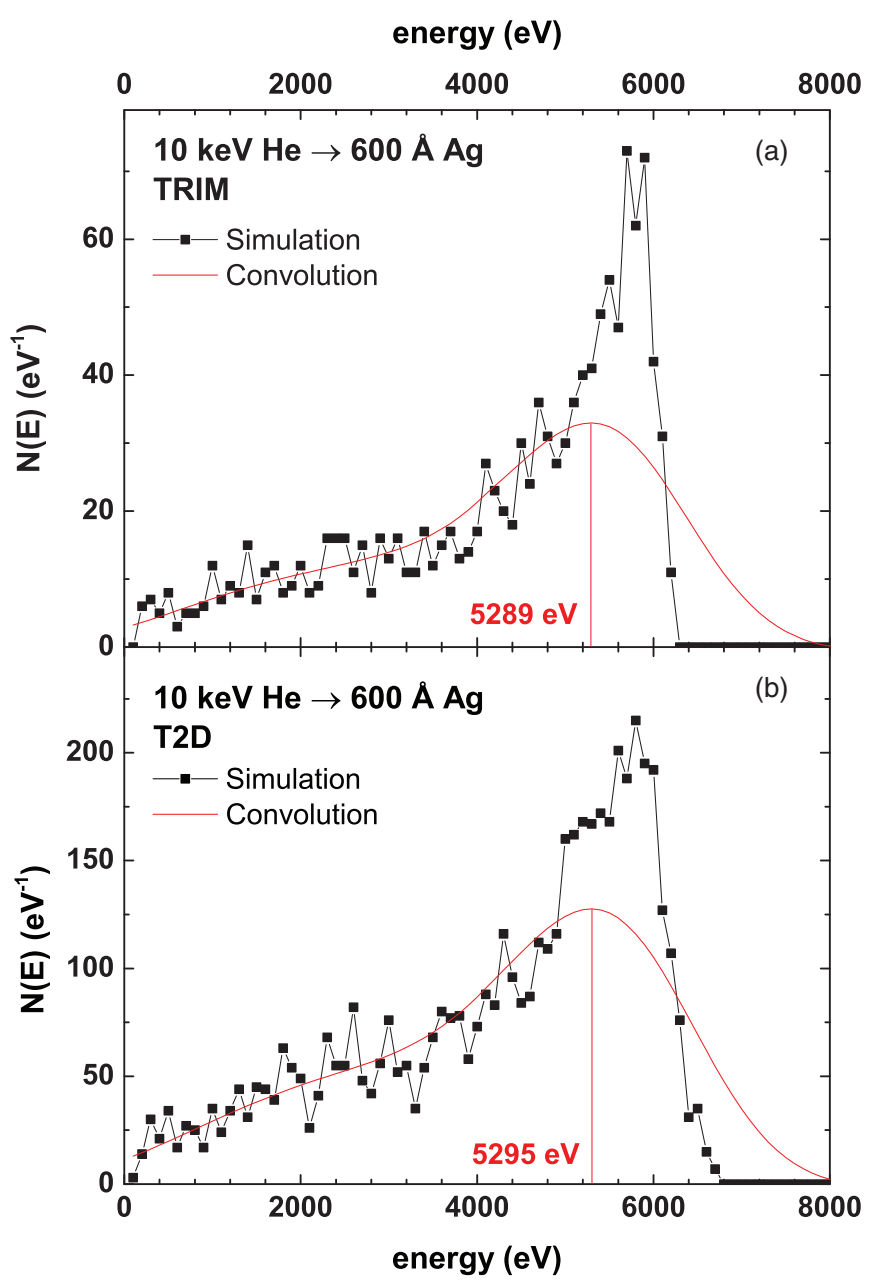

FIG. 2. (Color online) Energy spectra of $10 \mathrm{keV}$ He transmitted through a $600 \AA \mathrm{Ag}$ film as simulated with TRIM (a) and TRIM-T2D (b). Only projectiles with $\alpha<1^{\circ}$ were considered. Simulated spectra (squares) were convoluted with a Gaussian (red line) to consider energy loss straggling. 

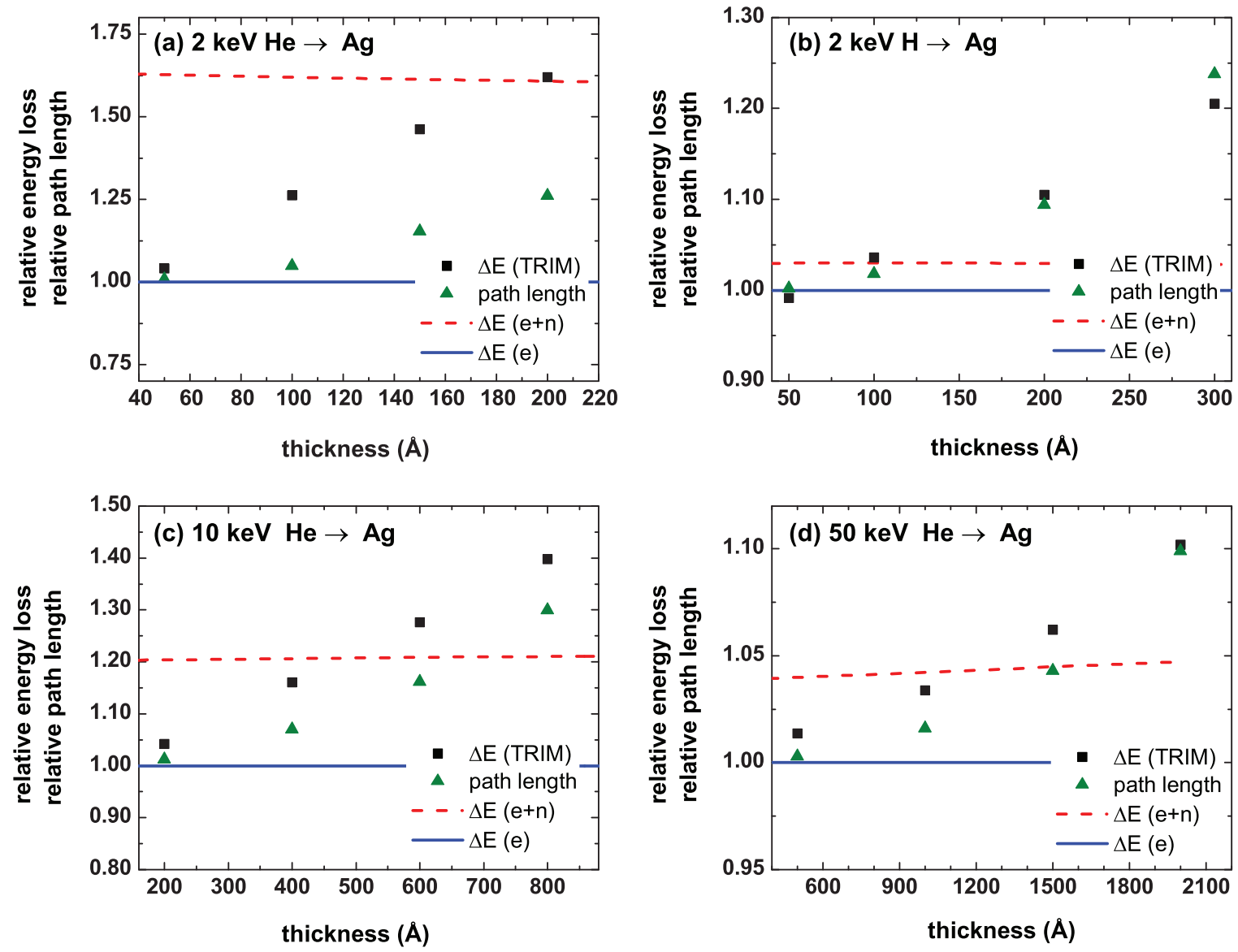

FIG. 3. (Color online) Results of TRIM simulations for He projectiles transmitted through Ag films of various thicknesses. Simulations were performed for $2 \mathrm{keV}$ (a), $10 \mathrm{keV}$ (c), and $50 \mathrm{keV}$ (d). Additionally, results are shown for $2 \mathrm{keV} \mathrm{H}$ (b). Only projectiles with $\alpha$ below $1^{\circ}$ or $2^{\circ}$, respectively, were considered. The most probable energy loss (squares) and the path length increase (triangles) were calculated for different thicknesses. The blue (continuous) and red (dashed) lines correspond to the energy loss due to electronic stopping only, $\Delta \mathrm{E}(\mathrm{e})$, and electronic + nuclear stopping, $\Delta \mathrm{E}(\mathrm{e}+\mathrm{n})$, respectively, for the nominal target thickness according to TRIM values. All energy loss values are given as ratios with respect to the energy loss for electronic stopping only; the path length is given relative to the nominal thickness.

is considered along the path, and impact parameter-dependent electronic losses are neglected. Along the trajectories, multiple atomic collisions are considered explicitly, and the corresponding energy loss due to recoil and the deflections are taken into account. Both programs yield the angular distribution of transmitted projectiles, $N(\alpha)$, and energy spectra for a given interval of transmission angles $\left[\alpha_{1}, \alpha_{2}\right]$. In addition, the TRIM-85 version allows us to store the path length distributions, $N(L, \alpha)$, and scattering angle distributions $N(\psi$, $\alpha$ ), for a given direction, $\alpha \in\left[0^{\circ}, 1^{\circ}\right]$ or $\alpha \in\left[0^{\circ}, 2^{\circ}\right]$ in our case. The relative contributions of $\Delta E_{n}^{*}$ and $\Delta E_{\mathrm{MS}}^{*}$ are deduced by comparing the $\mathrm{MC}$ results to the expected electronic energy loss along a straight line trajectory $\left(\Delta E_{e}\right)$.

For BS geometry, the TRIM for Rutherford backscattering spectroscopy (TRBS) code is used which allows for multiple collisions and electronic stopping along the trajectory [43]. In order to reduce computing time, only perpendicular incidence is considered, and for a chosen interval of scattering angles $\left[\theta_{1}, \theta_{2}\right]$, scattered projectiles are collected for $2 \pi$ azimuth acceptance. These TRBS simulations yield the energy spectra of backscattered ions, $N\left(E_{f}\right)$. Path length distributions and scattering angle distributions are again calculated with the
TRIM-85 code. The importance of nuclear losses and path length increase is investigated by comparing the MC results to calculations with the single scattering model, using the same interaction potential (universal potential) [42].

\section{RESULTS AND DISCUSSION}

\section{A. Transmission}

To illustrate our evaluation procedure, TRIM-85 and TRIMT2D spectra are shown in Fig. 2 for $10 \mathrm{keV} \mathrm{He}$ transmitted through a $600 \AA \mathrm{Ag}$ film. In these spectra $\alpha$ was restricted to $0^{\circ}-1^{\circ}$. Electronic energy loss straggling was considered by convoluting the resulting spectra with a Gaussian (red line). The magnitude of straggling was approximated based on the results in Ref. [44]. Since especially for low energies and large foil thicknesses the energy loss distributions are very broad, no attempt was made to evaluate the mean energy loss $\langle\Delta E\rangle$. Instead, the most probable energy loss, $\Delta \hat{\mathrm{E}}$, was determined by taking the maximum of the energy loss distribution. In this case, the most probable energy loss was determined to $\sim 4710$ $\mathrm{eV}$. Both spectra lead to concordant results, giving confidence in the chosen simulation parameters. 


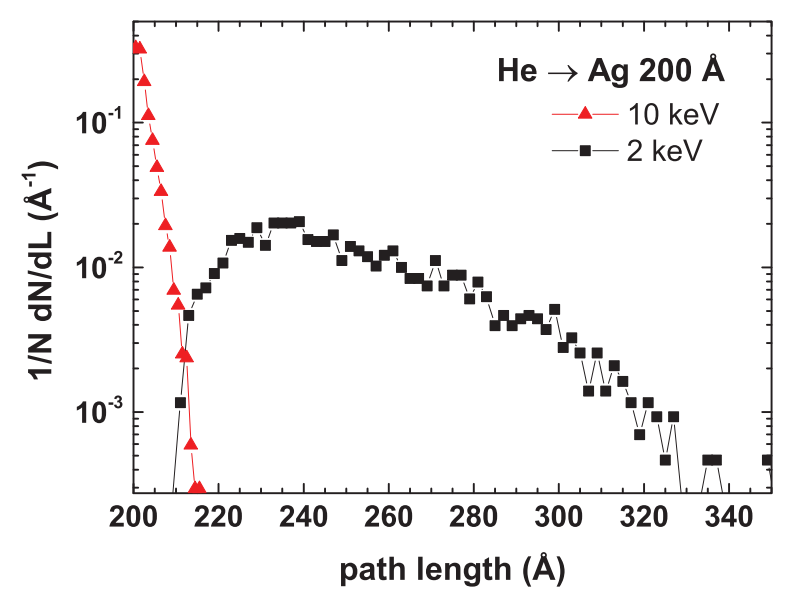

FIG. 4. (Color online) Path length distribution for He projectiles transmitted through a Ag film of $200 \AA$ thickness for different primary energies: $10 \mathrm{keV}$ (triangles) and $2 \mathrm{keV}$ (squares). Only projectiles with an exit angle of $0^{\circ}-1^{\circ}(10 \mathrm{keV})$ or $0^{\circ}-2^{\circ}(2 \mathrm{keV})$ and an exit energy within $1 \sigma$ (according to broadening due to straggling) of the most probable energy loss were considered. Simulations were performed with TRIM.

Simulations were performed for He ions with a primary energy of 2,10, and $50 \mathrm{keV}$ and $\mathrm{Ag}$ foils of various thicknesses (from 5 to $200 \mathrm{~nm}$ ). For each energy, target thicknesses were chosen such that projectiles transmitted in $0^{\circ}-1^{\circ}$ either lost up to $70 \%$ of their primary energy or were so low in intensity due to MS that it was not possible to evaluate the energy loss with sufficient precision. The selected energies cover a wide range of $S_{n} / S_{e}$ values, i.e., $0.04 \leqslant S_{n} / S_{e} \leqslant 0.5$. Complementary calculations were performed for $2 \mathrm{keV} \mathrm{H}$ and $\mathrm{Ag}$ foils in the range 5 to $30 \mathrm{~nm}$. From the resulting spectra, the most probable energy loss $\Delta \hat{E}$ was evaluated.

The evaluations are presented in Fig 3. To allow for a better comparison, $\Delta \hat{E} / \Delta E_{0}$ is depicted, where $\Delta E_{0}$ refers to the hypothetical electronic energy loss (using TRIM stopping) of a projectile traversing the target without elastic scattering. These plots also contain the relative mean path length of the transmitted projectiles, $\langle L(\alpha)\rangle / \Delta x$. The following qualitative results were obtained: at sufficiently high energies or sufficiently small foil thicknesses, respectively, the probability for violent atomic collisions is very low. Consequently, nuclear stopping and MS are negligible in this regime, $\Delta E_{\mathrm{tot}}=\Delta E_{e}=$ $\Delta E_{0}$, and the mean path length is equal to the foil thickness. At energies that are too low or foil thicknesses that are too large, respectively, nuclear stopping and MS contribute significantly and $\Delta E_{\text {tot }}>\Delta E_{e}$. Note that this behavior is observed for all energies, i.e., independent of the relative importance of nuclear stopping. For a given foil thickness, however, the largest deviations from $\Delta E_{0}$ are obtained at the lowest energy. For higher energies, where $S_{n} \ll S_{e}$, one can observe that the path length increase poses the dominant contribution to the increase in energy loss. This indicates that the influence of MS dominates over $S_{n}^{*}$; therefore $\Delta E_{\text {total }}>\Delta E_{e}+\Delta E_{n}$ is possible for sufficiently high values of $\psi_{1 / 2}$. Path length distributions for two selected cases are shown in Fig. 4, i.e., for 2 and $10 \mathrm{keV} \mathrm{He}{ }^{+}$ions transmitted through a $200 \AA \mathrm{Ag}$ target. Only projectiles are considered which exit the target with a final energy out of the interval $[\hat{E}-\sigma, \hat{E}+\sigma]$, where

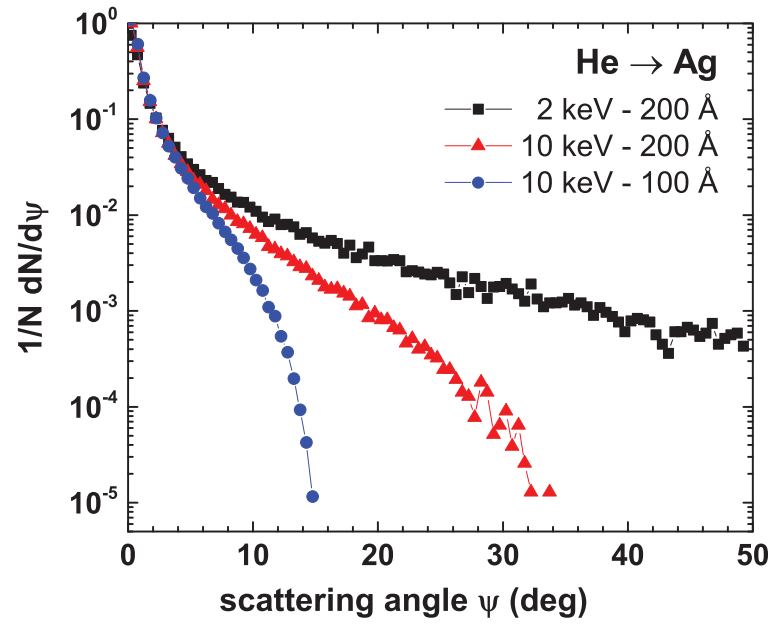

FIG. 5. (Color online) Scattering angle distribution for He projectiles transmitted through a Ag film with primary energy/film thickness of $2 \mathrm{keV} / 200 \AA$ (squares), $10 \mathrm{keV} / 100 \AA$ (circles), and $10 \mathrm{keV} / 200 \AA$ (triangles). Only projectiles with an exit angle of $0^{\circ}-1^{\circ}(10 \mathrm{keV})$ or $0^{\circ}-2^{\circ}(2 \mathrm{keV})$ and an exit energy within $1 \sigma$ (according to broadening due to straggling) of the most probable energy loss were considered. The distributions are based on the individual scattering events for trajectories in TRIM simulations.

$\sigma$ denotes the electronic straggling (standard deviation). The relative mean path lengths, plotted in Fig. 3, were deduced from such distributions.

The corresponding scattering angle distributions are presented in Fig. 5. These distributions are based on the individual scattering events for trajectories in the TRIM simulations. As expected, the smallest scattering angles (corresponding to the largest impact parameters) prevail, with a probability that depends only weakly on the simulation parameters (energy, thickness). The relative importance of larger scattering angles increases with increasing scattering probability, i.e., at lower energies and larger thicknesses. The strong increase in the relative importance of longer path lengths, which are observed for $200 \AA$ thick Ag foils and $2 \mathrm{keV} \mathrm{He}$ compared to $10 \mathrm{keV}$ $\mathrm{He}$, can be traced back to the increased importance of large scattering angles at the lower energy (see Fig. 4).

\section{B. Backscattering}

TRBS simulations were performed for $\mathrm{He}$ ions with a primary energy of $10 \mathrm{keV}$, and $\mathrm{Ag}$ layers with thicknesses up to $20 \mathrm{~nm}$. For the simulations, we considered a scattering angle $\theta=129^{\circ}$ as in our experimental apparatus. In most of the cases $S_{n} \ll S_{e}$, and simulations in transmission geometry revealed only a little influence of PS and MS on $\Delta E_{\text {tot }}$. In Fig. 6, the TRBS simulations are compared to results obtained in calculations within the single scattering model (SSM). SSM simulations were performed using electronic stopping only and electronic plus nuclear stopping (dotted line and dash-dotted line, respectively). The spectra were normalized such that the plateau heights of TRBS simulations and SSM calculations coincide at $k E_{0}$. For all depicted spectra clear differences between TRBS spectra and the SSM can be seen. In the case of $40 \AA$, the plateau and the low-energy edge are in good agreement with the SSM, although already at this low 


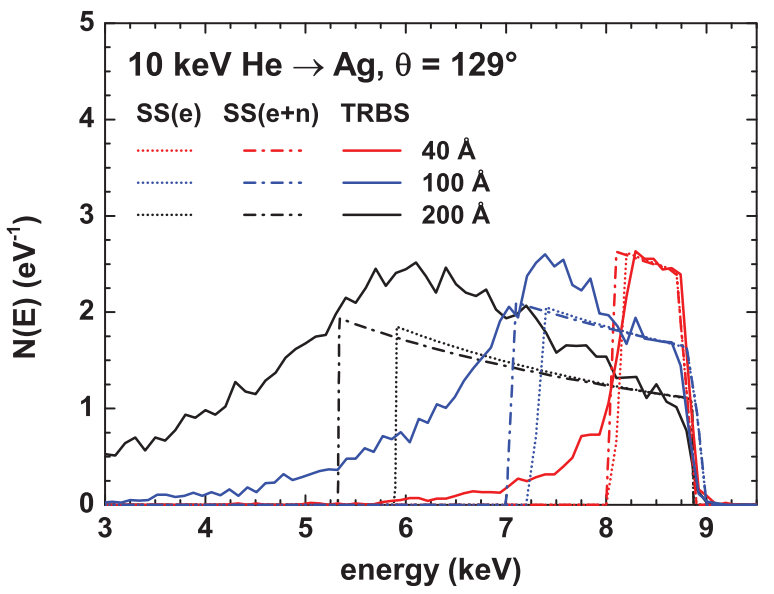

FIG. 6. (Color online) TRBS simulations of $10 \mathrm{keV} \mathrm{He}$ ions backscattered from thin Ag films. Simulations are compared to calculations based on the single-scattering model with electronic stopping only (dotted line) and electronic and nuclear stopping (dash-dotted line). For a given thickness, spectra were normalized to the high-energy edge. The offset of the different spectra at the high-energy edge was introduced for the sake of readability.

thickness, a noticeable tail is visible at energies below the low-energy edge. For larger thicknesses the low-energy edge is smeared out as well as a clear increase of the plateau height compared to the SSM is observed.

The importance of PS and MS in backscattering can easily be seen in scattering angle distributions as shown in Fig. 7. These distributions were obtained in TRIM simulations using the same code as for transmission. Similarly as in Fig. 5, the scattering angle distributions were evaluated from the individual scattering events in the TRIM trajectories; only trajectories were analyzed with a final energy within the single-scattering region. Figure 7(a) displays the scattering angle distribution for backscattering of $10 \mathrm{keV} \mathrm{He}$ from $\mathrm{Ag}$ films of different thicknesses. In Fig. 7(b) the scattering angle distribution is depicted for backscattering of He from $100 \AA$ $\mathrm{Ag}$ for energies from 2 to $50 \mathrm{keV}$. For a thickness of $3 \AA$ and 10 $\mathrm{keV}$, the SSM is fully confirmed. The relative importance of the contributions of PS and MS increases with decreasing energy and increasing thickness, while the relative importance of single scattering by an angle $\psi=\theta$ decreases. Additionally, a blurring of the peak corresponding to the large angle scattering $\psi=\theta$ due to small angle scattering is observed. Except for the $3 \AA$ case, the probability for small angle scattering prevails at all energies and thicknesses. For instance, for $2 \mathrm{keV} \mathrm{He}$ scattered from $100 \AA \mathrm{Ag}$, the probability for scattering by $\psi=40^{\circ}$ exceeds that for $\psi=\theta$ by a factor of $\sim 3.5$.

To make the qualitative differences between BS and TR clear it is helpful to compare the probability distributions Figs. 5 and 7 . For $\theta=129^{\circ}$, the ideal path length is $\sim 2.6$ $\times \Delta x$. It is, therefore, convenient to compare, e.g., $100 \AA$ in TR to $40 \AA$ in BS. For $10 \mathrm{keV} \mathrm{He}$, no scattering by angles above $15^{\circ}$ is observed for $100 \AA$ in TR, while for $40 \AA$ in BS all angles up to $150^{\circ}$ contribute with significant probability. This corroborates our earlier statement that TR is mainly influenced by small angle scattering, while in BS also collisions involving larger angles play a decisive role.
Within the SSM, the path length $L$ is in a one-to-one correspondence with the final energy $E$. Therefore, the energy spectrum, $d N / d E$, can be directly converted into a path length spectrum, $d N / d L$, via $d N / d L=(d N / d E) d E / d L$. For $10 \mathrm{keV} \mathrm{He}$ ions, the path length spectra within the SSM were numerically calculated for Ag films of a thickness of 40 and $200 \AA$, respectively. The results are displayed in Fig. 8 together with the path length distributions obtained from MC simulations including multiple scattering. For the evaluation of the MCsimulations, only trajectories with a final energy within the expected energy range based on the SSM were considered. Compared to the results from the SSM, PS and MS lead to an increase of the number of trajectories with a given path length, and trajectories with a path length longer than expected from the SSM. These features, however, are easily explained: If one allows the projectile to undergo more than just one single scattering event, the overall possibility to reach the detector is increased. Evidence for trajectories with a combination of large angle and small angle collisions which lead to a total scattering angle of $\theta=129^{\circ}$ can be seen in Fig. 7(a) when looking at the broadening of the single scattering peak. If the projectile undergoes two or more collisions with comparably large scattering angles, the nuclear energy loss is decreased
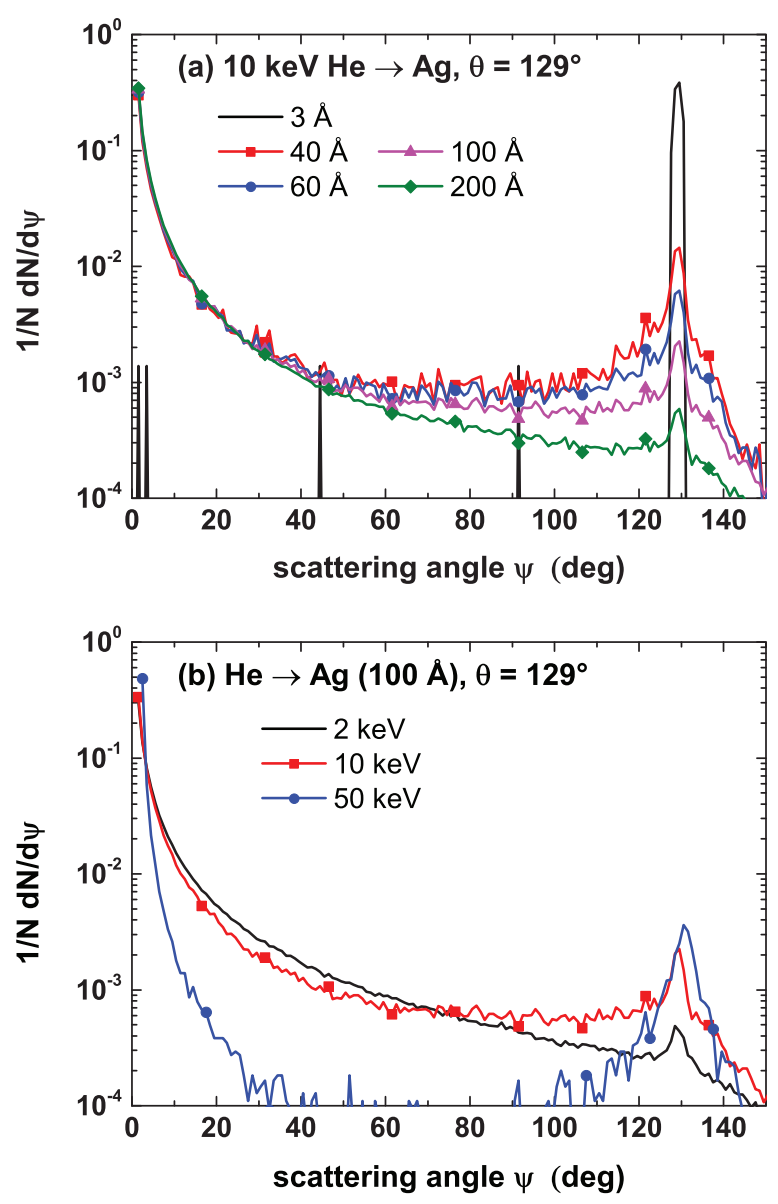

FIG. 7. (Color online) Scattering angle distributions for (a) $10 \mathrm{keV} \mathrm{He}$ ions scattered from $\mathrm{Ag}$ films of various thicknesses; (b) 2, 10, or $50 \mathrm{keV} \mathrm{He}$ ions scattered from a $100 \AA \mathrm{Ag}$ film. Results are based on the evaluation of individual scattering events of TRIM-based trajectories which have a final energy within the SSM. 


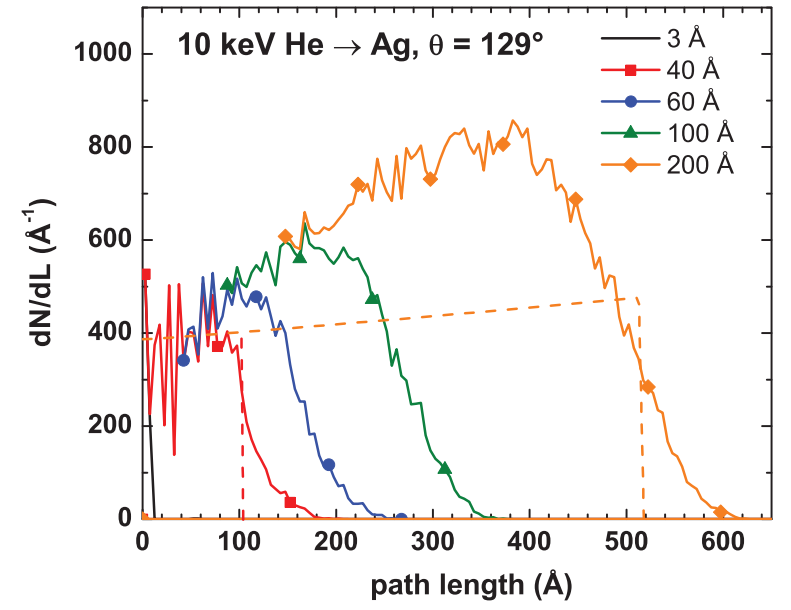

FIG. 8. (Color online) Path length distribution for $10 \mathrm{keV} \mathrm{He}$ scattered from $\mathrm{Ag}$ films of various thicknesses. The solid lines correspond to MC-simulations; the dashed lines are calculated using the single scattering model.

compared to a single large angle collision. This can explain the occurrence of trajectories with a final energy within the SSM window but a longer path length than expected.

\section{SUMMARY AND OUTLOOK}

The simulations showed that nuclear losses and MS may contribute to both TR and BS when the energy is very low and/or the target thickness is very large. In TR, the observed energy loss may exceed the value expected for electronic stopping along a straight line trajectory. In BS, more pronounced effects may be observed, especially due to the influence of collisions by larger angles. These observations indicate the need for adequate procedures to evaluate electronic stopping from measured spectra, e.g., by Monte Carlo simulations that allow for multiple atomic collisions.

Until now there has been no systematic study available which discusses whether for slow ions the different selection of impact parameters in TR and in BS may lead to a significant difference in the deduced stopping power values. For such a study, all other contributions must be known well enough so that observed differences in energy losses observed in TR and BS can be traced back to these processes.

\section{ACKNOWLEDGMENTS}

We thank Dr. Daniel Primetzhofer for inspiring discussions. Support by the Austrian Science Fund (FWF): Project P22587 is gratefully acknowledged. D. Goebl was supported by a DOC fellowship of the Austrian Academy of Sciences.
[1] E. Fermi and E. Teller, Phys. Rev. 72, 399 (1947).

[2] I. Nagy, A. Arnau, and P. M. Echenique, Phys. Rev. B 38, 9191 (1988).

[3] N. R. Arista and P. Sigmund, Phys. Rev. A 76, 062902 (2007).

[4] G. Martínez-Tamayo, J. C. Eckardt, G. H. Lantschner, and N. R. Arista Phys. Rev. A 54, 3131 (1996).

[5] E. Figueroa, E. Cantero, J. Eckardt, G. Lantschner, J. Valdés, and N. Arista, Phys. Rev. A 75, 064902 (2007).

[6] E. D. Cantero, G. H. Lantschner, J. C. Eckardt, and N. R. Arista, Phys. Rev. A 80, 032904 (2009).

[7] S. N. Markin, D. Primetzhofer, M. Spitz, and P. Bauer, Phys. Rev. B 80, 205105 (2009).

[8] M. A. Zeb, J. Kohanoff, D. Sanchez-Portal, A. Arnau, J. I. Juaristi, and E. Artacho, Phys. Rev. Lett. 108, 225504 (2012).

[9] M. Famá, G. H. Lantschner, J. C. Eckardt, N. R. Arista, J. E. Gayone, E. Sanchez, and F. Lovey, Nucl. Instrum. Meth. B 193, 91 (2002).

[10] M. Draxler, S. P. Chenakin, S. N. Markin, and P. Bauer, Phys. Rev. Lett. 95, 113201 (2005).

[11] L. N. Serkovic, E. A. Sánchez, O. Grizzi, J. C. Eckardt, G. H. Lantschner, and N. R. Arista, Phys. Rev. A 76, 040901(R) (2007).

[12] S. N. Markin, D. Primetzhofer, and P. Bauer, Phys. Rev. Lett. 103, 113201 (2009).

[13] M. Behar, R. C. Fadanelli, L. C. C. M. Nagamine, E. D. Cantero, G. H. Lantschner, J. C. Eckardt, N. R. Arista, R. Garcia-Molina, and I. Abril, Eur. Phys. J. D 66, 247 (2012).

[14] A. Dalgarno and G. W. Griffing, Proc. R. Soc. London A 232, 423 (1955).
[15] A. Arnau, M. Peñalba, P. Echenique, F. Flores, and R. Ritchie, Phys. Rev. Lett. 65, 1024 (1990).

[16] D. Primetzhofer, S. Rund, D. Roth, D. Goebl, and P. Bauer, Phys. Rev. Lett. 107, 163201 (2011).

[17] D. Primetzhofer, D. Goebl, and P. Bauer Nucl. Instrum. Meth. B (2012), doi: 10.1016/j.nimb.2012.12.091.

[18] W. Neuwirth, U. Hauser, and E. Kühn, Z. Phys. 220, 241 (1969).

[19] P. Mertens, Nucl. Instrum. Meth. B 27, 315 (1987).

[20] P. Bauer, Nucl. Instrum. Meth. B 27, 301 (1987).

[21] E. I. Sirotinin, A. F. Tulinov, A. Fiderkevich, and K. S. Shyshkin, Radiat. Eff. 15, 149 (1972).

[22] J. R. Tesmer and M. Nastasi, Handbook of Modern Ion Beam Materials Analysis (Materials Research Society, Pittsburgh, 1995).

[23] L. G. Glazov and P. Sigmund, Nucl. Instrum. Meth. B 207, 240 (2003).

[24] P. L. Grande and G. Schiwietz, Phys. Rev. A 44, 2984 (1991).

[25] E. A. Figueroa, E. D. Cantero, J. C. Eckardt, G. H. Lantschner, M. L. Martiarena, and N. R. Arista, Phys. Rev. A 78, 032901 (2008).

[26] A. Hentz, G. S. Parkinson, P. D. Quinn, M. A. Muñoz-Márquez, D. P. Woodruff, P. L. Grande, G. Schiwietz, P. Bailey, and T. C. Q. Noakes, Phys. Rev. Lett. 102, 096103 (2009).

[27] K. Khalal-Kouache, D. Primetzhofer, and P. Bauer, Nucl. Instrum. Meth. B 269, 1425 (2011).

[28] D. Goebl, D. Roth, and P. Bauer, Phys. Rev. A 87, 062903 (2013).

[29] D. Semrad, P. Mertens, and P. Bauer, Nucl. Instrum. Meth. B 15, 86 (1986). 
[30] N. E. B. Cowern, P. M. Read, C. J. Scofield, L. B. Bridwell, and M. W. Lucas, Phys. Rev. A 30, 1682 (1984), and references therein.

[31] H. Brongersma, M. Draxler, M. Deridder, and P. Bauer, Surf. Sci. Rep. 62, 63 (2007).

[32] P. Sigmund and B. Winterbon, Nucl. Instr. Meth. 119, 541 (1974); 125, 491 (1975).

[33] A. Weber, H. Mommsen, W. Sarter, and A. Weller, Nucl. Instrum. Meth. 198, 527 (1982).

[34] R. D. Geil, M. Mendenhall, R. A. Weller, and B. R. Rogers, Nucl. Instrum. Meth. B 256, 631 (2007).

[35] P. Bauer, E. Steinbauer, and J. P. Biersack, Nucl. Instrum. Meth. B 79, 443 (1993).

[36] G. Amsel, G. Battistig, and A. L'Hoir, Nucl. Instrum. Meth. B 201, 325 (2003).

[37] C. D. Archubi, J. C. Eckardt, G. H. Lantschner, and N. R. Arista, Phys. Rev. A 73, 042901 (2006).
[38] E. D. Cantero, G. H. Lantschner, J. C. Eckardt, F. C. Lovey, and N. R. Arista, Phys. Rev. A 81, 042902 (2010).

[39] M. Fama, G. H. Lantschner, J. C. Eckardt, C. D. Denton, and N. R. Arista, Nucl. Instrum. Meth. B 164, 241 (2000).

[40] J. P. Biersack and L. G. Haggmark, Nucl. Instrum. Meth. 174, 257 (1980).

[41] K. Khalal-Kouache, A. C. Chami, M. Boudjema, Y. Boudouma, B. Arezki, C. Benazeth, and P. Benoit-Cattin, Alg. J. Adv. Mater. 2, 87 (1998).

[42] J. F. Ziegler, J. P. Biersack, and U. Littmark, The Stopping and Range of Ions in Solids (Pergamon Press, New York, 1985).

[43] J. P. Biersack, E. Steinbauer, and P. Bauer, Nucl. Instrum. Meth. B 61, 77 (1991).

[44] S. P. Møller, A. Csete, T. Ichioka, H. Knudsen, H.-P. E. Kristiansen, U. I. Uggerhøj, H. H. Andersen, P. Sigmund, and A. Schinner, Eur. Phys. J. D 46, 89 (2008) 